\title{
PREVALENCIA DE Fasciola hepatica Y PÉRDIDAS ECONÓMICAS ASOCIADAS AL DECOMISO DE HÍGADOS EN TRES MATADEROS DE CLASE A DE COSTA RICA
}

\author{
Diana Rojas $^{1 / *}$, José Andrés Cartín $^{* *}$ \\ Palabras clave: Vigilancia epidemiológica; parasitología; trematodos; ganadería; diagnóstico. \\ Keywords: Epidemiological surveillance; parasitology; flukes; livestock; diagnosis.
}

Recibido: 09/03/16

\section{RESUMEN}

Para estimar la prevalencia anual y mensual de Fasciola hepatica y las pérdidas económicas anuales asociadas al decomiso de hígados por presencia del parásito, se analizaron los registros del Ministerio de Agricultura y Ganadería de Costa Rica, Dirección de Inocuidad de Productos de Origen Animal (DIPOA), para el 2014, provenientes de 3 mataderos clase A localizados en el área metropolitana de Costa Rica; en estos mataderos se sacrificaron 249108 reses, de las cuales 4547 hígados fueron decomisados por presencia de $F$. hepatica en el órgano. Las mayores prevalencias, entre 2,33 y $2,55 \%$, se presentaron en enero, febrero y marzo, y las menores, entre 1,32 y $1,56 \%$, durante agosto, setiembre y octubre. La prevalencia anual según estos registros fue 1,83\% (IC 95\%: 1,77-1,88). Las pérdidas económicas asociadas al decomiso de hígados fueron de 36.379.000 CRC, equivalentes a 67.438 USD. Se resalta el perjuicio económico de este parásito a nivel nacional y la utilidad del decomiso y registro de vísceras afectadas en los establecimientos de sacrificio de bovinos, como herramienta diagnóstica para la vigilancia epidemiológica, disponible para conocer sobre el estado de esta parasitosis. Como alternativas de control y prevención de la enfermedad, se plantea

1 Autora para correspondencia. Correo electrónico: diana.rojas_a@ucr.ac.cr

Universidad de Costa Rica, Facultad de Microbiología, Departamento de Parasitología, Centro de Investigación en Enfermedades Tropicales, Costa Rica.
Aceptado: 03/06/16 
considerar las condiciones específicas de las regiones afectadas y así controlar esta parasitosis con un manejo integral, que involucre la fuente de alimento, desparasitación regular del ganado, con registro y monitoreo farmacológico de las drogas utilizadas, y saneamiento ambiental en fincas afectadas.

\section{INTRODUCCIÓN}

En Costa Rica, la carne bovina ocupa el primer lugar como fuente de proteína de origen animal para el consumo humano, constituye el tercer recurso proteico de la dieta de los costarricenses y se asocia con una actividad productiva que genera ganancias para un gran sector de la población (Arronis 2004). Según el censo ganadero del 2014, a nivel nacional existen aproximadamente 1278817 cabezas de ganado, de las cuales un $42,1 \%$ se encuentran en la actividad de cría y engorde, un $32 \%$ en doble propósito y un $25,6 \%$ en producción lechera. Del total de fincas del país, un 28,51\% (26 516 fincas) declaran que su principal actividad es la ganadería vacuna (Morales y Murillo 2015).

El bienestar, la salud y el manejo del ganado destinado para sacrificio es un tema relevante, principalmente para la economía de Costa Rica, que está entre los principales exportadores de carne de Centroamérica. De la producción anual, se estima que 67-70 mil toneladas se consume internamente y 19741 toneladas se exportan y generan aproximadamente $\$ 47.261 .000$ USD por año (Barrantes et al. 2007). Es por esta y otras razones que cualquier tópico asociado con el detrimento de la salud en estos animales tiene impacto sobre la producción del sector pecuario y repercusiones en la economía del país.

Uno de los factores que afecta a estos animales son las parasitosis, ya sea por helmintos o protozoarios (Vintimilla 2013). En el manejo del of this parasitic disease. As alternatives for the control and prevention of $F$. hepatica, it is advisable to consider the specific conditions of the regions affected and thus control this parasitosis through integrated management, involving the food source and regular deworming of livestock, with registration and monitoring of drugs used for de-worming, and environmental sanitation at affected farms.

ganado bovino muchas de estas infecciones parasitarias son clasificadas como subclínicas, sin que esto signifique que no representen pérdidas económicas en los animales afectados. Dentro de las enfermedades parasitarias más importantes que afectan a este grupo se incluye la fasciolosis, enfermedad provocada por $F$. hepatica y considerada como la infección causada por helmintos más importante en bovinos de países tropicales (Abebe et al. 2010). En las reses, esta enfermedad es de lenta evolución y se caracteriza por pérdida de peso, emaciación, edema, debilidad, diarrea y ascitis (Tsegaye et al. 2012). Las tasas de morbilidad y mortalidad son variables, según la región geográfica de procedencia del ganado (OPS 2003).

Además de su importancia veterinaria en todo el mundo, la fasciolosis se ha catalogado recientemente como una zoonosis emergente ampliamente distribuida (Yildirim et al. 2007, Howell 2011) que se asocia principalmente a regiones donde la fasciolosis en rumiantes es endémica (Das Chagas et al. 2011). La enfermedad en humanos se manifiesta principalmente en el hígado, donde las secuelas patogénicas más importantes son lesiones hepáticas y fibrosis, acompañadas de inflamación crónica de los ductos biliares (Mas-Coma 2004).

Aunque difícil de cuantificar, la infección de más de 600 millones de rumiantes domésticos con $F$. hepatica a nivel mundial, causa pérdidas económicas cercanas a los $\$ 200$ millones por año en el sector agrícola mundial (Becerra 2001, 
Bennema et al. 2011, Martínez et al. 2012). Estas pérdidas económicas varían dentro de una misma zona geográfica o dentro de la misma finca en los diferentes meses o años; y dependen de la interacción entre los aspectos fisiopatológicos de la enfermedad y los ambientales (factores climatológicos y geográficos) que determinan la presencia de hospederos intermediarios y el parásito en el ambiente (OPS 2003). Las pérdidas económicas más relevantes son las que ocurren por el decomiso de órganos afectados, muerte de los animales en los casos severos y una disminución en la eficiencia productiva de leche y carne de hasta un 20-30\% (Gorman et al. 1998).

Esta situación es difícil de controlar debido a la complejidad del ciclo de vida del parásito $\mathrm{y}$ numerosos factores de riesgo que involucran desde condiciones ambientales y climáticas, presencia del hospedero intermediario (Howell et al. 2012), hasta voluntad política de control de la infección en granjas bovinas o desparasitación regular de los hospederos naturales (Yildirim et al. 2007, Fromsa et al. 2011).

En el caso de Costa Rica, el Servicio Nacional de Salud Animal (SENASA), amparado bajo la Ley $N^{\circ} .8495$ (Ley SENASA) y basado en el Reglamento Sanitario y de Inspección Veterinaria de Mataderos, Producción y Procesamiento de Carnes No. 29588-MAG-S, se desempeña por medio de la inspección oficial, asignada a los establecimientos de sacrificio de bovinos, como un tamiz sanitario en las plantas, disminuyendo en gran medida aquellas enfermedades presentes en los animales durante su sacrificio y procesamiento. La inspección oficial se encarga de identificar si los animales son aptos para consumo humano o no y por lo tanto determinar si se procede con el decomiso total de la canal o alguna parte de esta. En el capítulo XXI del decreto No. 29588MAG-S se establece como causa de decomiso del hígado en su totalidad, la presencia del parásito $F$. hepatica en este órgano (Poder Ejecutivo 2001). En concordancia con esto, el Ministerio de Agricultura y Ganadería (MAG), específicamente SENASA en la Dirección de Inocuidad de Productos de Origen Animal (DIPOA), lleva un registro sobre los decomisos en mataderos de res de los hígados infectados por este parásito (DIPOA 2012).

Pocos estudios han sido llevados a cabo alrededor de esta parasitosis en Costa Rica, y los efectuados señalan su presencia en la provincia de Cartago (Chavarría 1939, Alice 1978, Herrera 1979, Chang y Cartín 1983) y Limón (Alpízar 2008). Algunas investigaciones llevadas a cabo en plantas de sacrificio y a partir de datos registrados sobre decomiso de hígados, se han revelado distintos valores de prevalencia de este parásito tales como: $1,3 \%$ al incluir los decomisos para setiembre, octubre, noviembre y diciembre de 1938 en un matadero nacional (Chavarría 1939), 3,4\% que contempla los datos de enero de 1976 a enero de 1977 en un matadero en Turrialba (Alice 1978) y $0,8 \%$ junto con datos comprendidos entre el 2000 y el 2006 en 3 mataderos del área metropolitana (Alpízar 2008).

Con la finalidad de estimar la prevalencia y las pérdidas económicas anuales asociadas al decomiso de hígados por la presencia de F. hepatica, se analizaron los registros compilados por DIPOA para 2014 provenientes de 3 mataderos de clase A localizados en el área metropolitana de Costa Rica.

\section{MATERIALES Y MÉTODOS}

Se estimó la prevalencia de $F$. hepatica, y sus intervalos de confianza al 95\%, a partir de los registros compilados por DIPOA-SENASA provenientes de los datos de decomiso de hígados por presencia de este parásito en 3 de los mataderos de clase A ubicados en el área metropolitana para el 2014. Este parámetro se obtuvo al dividir el número total de hígados decomisados entre el número total de reses sacrificadas, en los 3 establecimientos durante el 2014. Estos mataderos cumplen con las regulaciones nacionales, están aprobados por terceros para la exportación y sacrifican hembras, machos y terneros provenientes de todo el país. Del total de reses sacrificadas en el país, un $82 \%$ se procesan en estos mataderos (Díaz et al. 2013), por lo que se estima que estos 
registros pueden dar un reflejo aproximado de la situación de esta parasitosis a nivel nacional.

En el documento DIPOA- PG-013 de SENASA, se estandariza la metodología que debe ser empleada por los médicos veterinarios inspectores y sus inspectores auxiliares oficiales en los establecimientos de sacrificio de origen bovino, para realizar las inspecciones post mortem; así como los criterios para la realización de los decomisos o retenciones de animales y sus derivados cárnicos. Concretamente, en el caso de la inspección del hígado, indica que éste debe colocarse con la parte visceral hacia arriba, para poder incidir los linfonodos hepáticos, haciéndole la mayor cantidad de incisiones posibles; y también que se deben incidir los ductos biliares en busca de cualquier patología o parásito. Finalmente se debe voltear para examinar, palpar y de ser necesario incidir, la parte parietal del mismo para lograr de esta forma el diagnóstico y decisión final de la víscera (DIPOA 2012).

Para estimar las pérdidas económicas debido al decomiso de esta víscera, se calculó el precio promedio en colones de un hígado de res al momento de la investigación y ese monto se multiplicó por el número de hígados decomisados durante el 2014. Los datos suministrados por el DIPOA sobre el decomiso mensual y anual en los 3 establecimientos durante el 2014, permitió visualizar la pérdida anual debida a esta causa y su comportamiento mensual.

\section{RESULTADOS Y DISCUSIÓN}

\section{Prevalencia}

Para el 2014, en 3 de los mataderos de clase A ubicados en el área metropolitana de Costa Rica se sacrificaron un total de 249108 reses; de las cuales 4547 hígados fueron decomisados por presencia de $F$. hepatica en la víscera (Figura 1). Las mayores prevalencias se presentaron en enero, febrero y marzo, mientras que las menores fueron en agosto, setiembre y octubre. La prevalencia anual obtenida a partir de estos registros fue de 1,83 (IC 95\%: 1,77 - 1,88) (Cuadro 1).
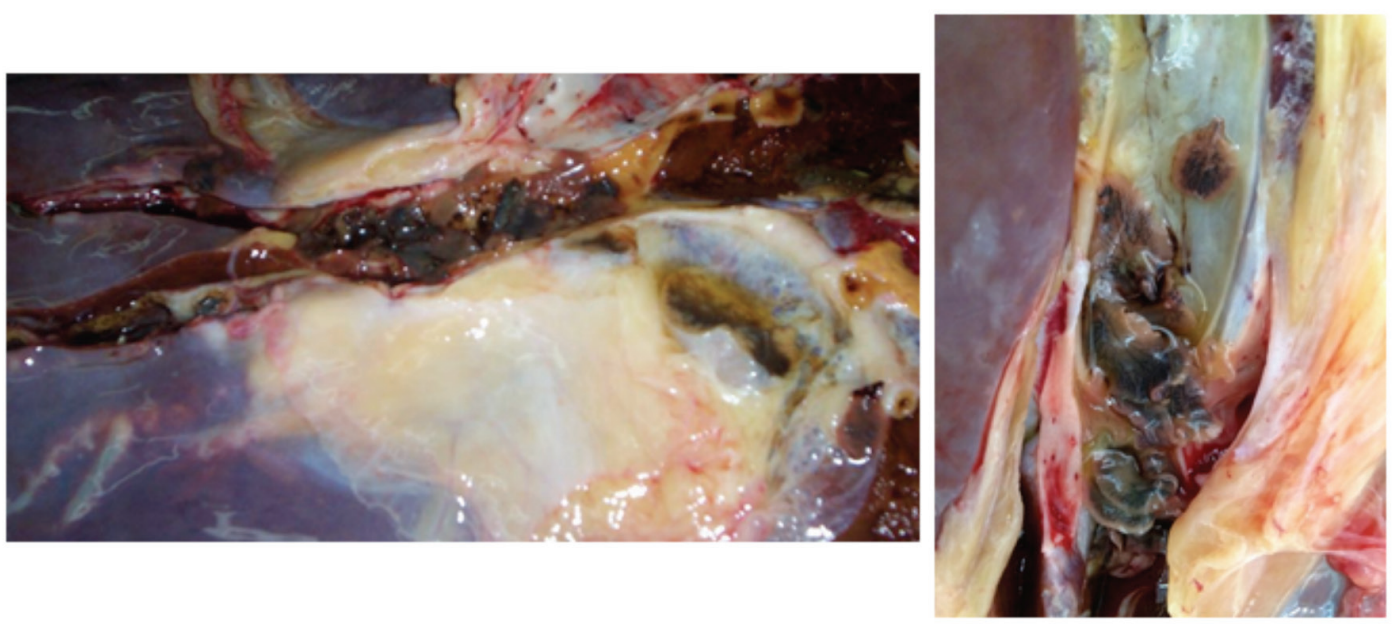

Fig. 1. Hígado decomisado por la presencia de Fasciola hepatica en la víscera (señalada con flechas). 
Cuadro 1. Prevalencia mensual y anual de F. hepatica con base en el registro de reses sacrificadas e hígados decomisados en 3 mataderos de clase A de Costa Rica, durante el 2014.

\begin{tabular}{lccc}
\hline Mes & Reses sacrificadas & Hígados decomisados & Prevalencia (I.C. $95 \%)$ \\
\hline Enero & 20530 & 478 & $2,33(2,12-2,53)$ \\
Febrero & 19336 & 499 & $2,58(2,36-2,80)$ \\
Marzo & 20005 & 511 & $2,55(2,34-2,77)$ \\
Abril & 17009 & 333 & $1,96(1,75-2,17)$ \\
Mayo & 17368 & 355 & $2,04(1,83-2,25)$ \\
Junio & 17197 & 279 & $1,62(1,43-1,81)$ \\
Julio & 22483 & 371 & $1,65(1,48-1,82)$ \\
Agosto & 299 & $1,32(1,18-1,47)$ \\
Septiembre & 245 & $1,08(0,95-1,22)$ \\
Octubre & 22572 & 365 & $1,56(1,40-1,72)$ \\
Noviembre & 23349 & 421 & $1,83(1,65-2,00)$ \\
Diciembre & 23031 & 391 & $1,66(1,49-1,82)$ \\
\hline Total & 23592 & 4547 & $1,83(1,77-1,88)$ \\
\hline
\end{tabular}

* I.C. $95 \%$ : Intervalo de confianza al $95 \%$.

El comportamiento mensual de decomisos encontrado en este estudio coincide con lo reportado por Alice (1978), en que los meses de mayor decomiso de hígados en un matadero en Turrialba también fueron enero, febrero y marzo; aunque no así con lo reportado por Alpízar et al. (2013), quienes tras recolectar muestras de hígados bovinos infectados con el parásito en 3 mataderos del Valle Central de agosto del 2005 a agosto del 2006, obtuvieron que los mayores porcentajes de infección en ese intervalo se dieron en agosto de ambos años. La falta de estudios que revelen el comportamiento mensual de decomiso a través de varios años, dificulta la visualización certera del comportamiento de esta enfermedad en las zonas afectadas y retrasa la implementación de programas de prevención y tratamiento dirigido en campo, ya que se desconoce realmente cuáles son los meses en los que es más probable que las reses se infecten en el país y porqué. La tendencia observada durante 12 meses no es suficiente para dilucidar el comportamiento de la enfermedad, ya que ésta puede ser influenciada por factores climáticos u otras condiciones que pueden asociarse con el establecimiento y propagación del parásito y que varíen entre año y año, de ahí la importancia de analizar el comportamiento que incluya un periodo apropiado que permita estimar una frecuencia promedio de los casos. De igual forma, los valores de prevalencia reportados en Costa Rica a partir de registros de decomisos de hígados en las plantas de sacrificio no han sido uniformes. En una investigación realizada por Chavarría (1939) se reportó una prevalencia cercana al $1,3 \%$ en un matadero nacional para setiembre, octubre, noviembre y diciembre de 1938. En el mismo documento de Alice (1978), se reportó una prevalencia de 3,4\% de enero de 1976 a enero de 1977 en un matadero en Turrialba; y en el análisis realizado por Alpízar (2008), en el cual se estudiaron los registros de decomiso de hígados infectados con el parásito en 3 mataderos del área metropolitana, durante el 2000 y el 2006, se reportó una prevalencia total de $0,8 \%$. 
Un aspecto interesante puntualizado en este último estudio es que del 2000 al 2006 se perfiló una tendencia anual hacia el aumento de decomisos de hígados en las plantas, por lo que habría que analizar si esta propensión al aumento se debió a una mejora en el sistema de detección del parásito y mejor capacitación del personal que hace la inspección, una mejora en el sistema de captura y sistematización de los datos, o un aumento de casos del parásito a nivel de las zonas en donde se mantiene el ciclo biológico. Una o varias de estas posibles razones podrían apoyar el hallazgo de un aumento en el valor de la prevalencia encontrado en el 2014, en comparación con el reportado en el 2000-2006, que es el estudio más comparable, en términos temporales y metodológicos.

Por lo reportado por otras investigaciones hechas en el país y la experiencia de veterinarios oficiales de SENASA y regentes en los mataderos, la infección por este parásito parece no ser homogénea en el país; es por esto que la prevalencia obtenida a partir del análisis global de los sacrificios y decomisos totales en uno o varios establecimientos, sin considerar la región geográfica de la que procede la res, es probable que sea menor a la presente en las zonas donde la fasciolosis es endémica. Esto puede verse reflejado en algunas de las investigaciones, tal y como la realizada por Herrera en 1979, donde se reporta un $17 \%$ de infección por $F$. hepatica en bovinos de carne de una finca en Agua Caliente de Cartago; o la realizada por Chang y Cartín en 1983, donde determinaron un 42\% de infección en bovinos de leche en fincas de Santa Cruz, cantón de Turrialba. De igual forma, Alpízar et al.
(2013) encontraron que durante agosto del 2005 a agosto del 2006, el porcentaje de infección en una finca en Siquirres de Limón fue de 11,26\%; y posteriormente, Rojas en el 2015 determinó que la prevalencia basada en decomiso de hígados infectados por F. hepatica en 3 mataderos de clase A del área metropolitana en setiembre y octubre del 2014 fue de 3,88\% para reses que exclusivamente provenían de la región Metropolitana y Huetar Atlántica, y de 0,5\% para reses provenientes de las otras regiones del país (Rojas 2015).

Todos estos hallazgos permiten suponer la presencia endémica de este parásito en ciertas zonas del país y por ende, resaltan la importancia de enfocar los esfuerzos de investigación en las áreas problema y trabajar con los ganaderos o personal relacionado al sector pecuario de zonas prioritarias de atención. Esta vigilancia regionalizada no sólo tiene importancia en términos de salud pública y logística; si no también en términos económicos, ya que es posible que sea un grupo de productores los más afectados por esta condición.

\section{Estimación de pérdidas económicas por decomiso de hígados}

Si se considera solamente el dato de pérdidas asociadas al decomiso de hígados, para el 2014 , se perdieron $\$ 36.379 .000$ colones, equivalentes a \$67.438 USD (Cuadro 2); dato que habría que analizar si tiene el mismo impacto al contemplar todas las regiones geográficas del país o si sólo incluyera las regiones que se sospecha son foco para la enfermedad. 
Cuadro 2. Pérdidas económicas asociadas al decomiso de hígados por la presencia de $F$. hepatica, en 3 mataderos de clase A de Costa Rica, durante el 2014.

\begin{tabular}{lr}
\hline Estimaciones económicas & 5 \\
\hline Peso promedio de 1 hígado de res (kg) & $\varnothing 1.600$ \\
Precio aproximado de $1 \mathrm{~kg}$ de hígado de res (colones) & $\varnothing 8.000$ \\
Precio promedio 1 hígado (colones) & 4547 \\
Total de hígados decomisados 2014 & $\varnothing 36.376 .000$ \\
Pérdida económica total por decomiso hígados (colones) & $\$ 67.438$ \\
\hline
\end{tabular}

* Tipo de cambio promedio compra venta, al último día del 2014 (Barquero y Muñoz 2015).

Las pérdidas económicas asociadas a esta parasitosis no sólo se relacionan con el decomiso de hígados parasitados en los mataderos del país, sino también a la reducción de peso, fertilidad, producción de leche, tratamiento, suplementos alimentarios y horas de trabajo del personal (Schweizer et al. 2005, Espinoza et al. 2010, Monteiro et al. 2013). Aunque estos datos aún no han sido estimados para Costa Rica, diversos estudios, como el llevado a cabo por Sánchez y Fraser (2013), señalan que la presencia del parásito se asocia a una reducción de al menos $0,3 \%$ en el precio de la canal de la res, ya que se relaciona con una reducción en el peso, contenido de grasa y músculo del animal. Otra perspectiva es la aportada por Kaplan (2001), el cual señala que la ganancia por un control de la fasciolosis en vacunos, en una encuesta extensa de ranchos realizado en Florida Estados Unidos, resultó en que el beneficio del control de este parásito traía consigo una ganancia de peso entre 6,2 y $10 \mathrm{~kg}$ en vacunos de carne, un aumento entre 1 a $3 \%$ más de crías y un incremento en el peso al destete en un rango de 13,6 a $20 \mathrm{~kg}$ (Kaplan 2001).

Para tratar de disminuir estos costos, se deberían contemplar programas de control bien elaborados para cada región, donde se tome en cuenta la epidemiología de la enfermedad en los bovinos, la variación estacional de las poblaciones de los caracoles, así como la estacionalidad de la infección por formas intermedias y las condiciones climáticas. Se deben considerar las condiciones específicas de cada zona, y probablemente la mejor forma de controlar esta parasitosis es a través de un manejo integral, que involucre la fuente del alimento para el pastoreo de las vacas, desparasitación regular del ganado (Morphew et al. 2007) y saneamiento ambiental, principalmente a través de una limitación y drenaje de los hábitats potenciales para el hospedero intermediario (Morales y Pino 2004, Howell 2011). Malone (2002) indica que la mayoría de las infecciones ocurren por el pastoreo en zonas que tienen o están cerca de hábitats para moluscos que no son fijos y por lo menos permanecen húmedas durante la mitad del año. Es importante suponer que para garantizar la sostenibilidad de un eventual programa, se debe contar con un registro eficiente y monitoreo farmacológico de la calidad de las drogas que se utilizan y la posible presencia de animales refractarios al tratamiento (Espinoza et al. 2010). De igual manera, por el carácter zoonótico de la enfermedad, es necesario trabajar con la población humana de zonas aledañas a sitios donde se sabe hay transmisión del parásito (Monteiro et al. 2013).

Particularmente, en Costa Rica sería conveniente poder enlazar el Sistema de Rastreabilidad 
de Ganado Bovino (sistema de control vigente y obligatorio para todo el territorio nacional desde el 2010), con el registro de decomisos centralizado por SENASA y otros datos importantes de los cuales disponen los establecimientos de sacrificio, tales como el sexo, edad, conformación del animal (peso, grasa y músculo), ya que unidos estos datos en una misma base de datos general, permitiría dar seguimiento y análisis no sólo a la fasciolosis, sino también a otras enfermedades monitoreadas en los mataderos que poseen un impacto en la salud pública animal y humana. Debido a que este sistema de rastreabilidad permite conocer con un grado aceptable de exactitud la procedencia de todos los bovinos que se movilizan o comercializan a nivel nacional desde el origen del embarque hasta la planta de sacrificio o faenado (Díaz et al. 2013), el enlace de datos propuesto permitiría dar rastreo a las fincas problema y así brindar un acompañamiento para controlar esta enfermedad de manera puntual, distribuyendo de manera más adecuada los recursos y acciones para contener, reducir y eliminar los riesgos potenciales a la salud y pérdidas asociadas a esta parasitosis. De igual forma, sería adecuado aprovechar todos los registros de los cuales DIPOA dispone por reglamento y generar boletines anuales sobre el estado de la enfermedad, que informen no sólo a los relacionados con la parte de salud animal, sino también a las autoridades de salud pública, ya que en la actualidad este decomiso y registro es la herramienta epidemiológica de la cual dispone el país para conocer sobre el estado de esta parasitosis. Esto facilitará la toma de decisiones respecto al establecimiento de planes y programas conjuntos para el combate de esta zoonosis y permitiría a SENASA implementar medidas de evaluación de riesgo para establecer normas sanitarias y direccionar los esfuerzos de la Institución para el control y erradicación de estas enfermedades vinculadas con animales; así como la inversión en capacitación y continua mejora técnica de sus veterinarios e inspectores oficiales en plantas de sacrificio, ya que son la pieza fundamental que logra filtrar la evidencia de la presencia de esta enfermedad en el país.

\section{LITERATURA CITADA}

Abebe, R; Abunna, F; Berhane, M; Mekuria, S; Megersa, B; Regassa, A. 2010. Fasciolosis: Prevalence, financial losses due to liver condemnation and evaluation of a simple sedimentation diagnostic technique in cattle slaughtered at Hawassa Municipal abattoir, southern Ethiopia. Ethiopian Veterinary Journal 14(1):39-51.

Alice, E. 1978. Fasciolosis Humana y Bovina (Datos Relativos a Fasciolosis Humana y Bovina en Costa Rica). Revista Médica de Costa Rica XLV (464):117-120.

Alpízar, C. 2008. Infección por Fasciola hepatica en bovinos de carne de una finca en Siquirres e impacto económico del decomiso de hígados en tres mataderos del área metropolitana de Costa Rica. Tesis Lic. Heredia, Costa Rica, Universidad Nacional. 40 p.

Alpízar, C; Bianque De Oliveira, J; Jiménez, A; Hernández, J; Berrocal, A; Romero, J. 2013. Fasciola hepatica en ganado bovino de carne en Siquirres y lesiones anatomo-histopatológicas de hígados bovinos decomisados en mataderos de Costa Rica. Agronomía Costarricense 37(2):7-16.

Arronis, V. 2004. Recomendaciones sobre sistemas intensivos de producción de carne: Estabulación, semiestabulación y suplementación estratégica de pastoreo. Ministerio de Agricultura y Ganadería (en línea). San José, Costa Rica. 24 p. Consultado 07 ene. 2015. Disponible en http://www.mag.go.cr/ biblioteca_virtual_animal/estabulacion.pdf

Barquero, J; Muñoz, E. 2015. Costa Rica: tipo de cambio real y zona de equilibrio. Banco Central de Costa Rica. Departamento de Investigación Económica. División Económica (en línea). San José, Costa Rica. 27 p. Consultado 18 ago. 2016. Disponible en http://www.bccr.fi.cr/investigacioneseconomicas/ politicacambiariaysectorexterno/Costa\%20Rica tipo_de_cambio_real_y_zona_de_equilibrio.pdf

Barrantes, J; Jiménez, R. 2007. Caracterización de la Agrocadena de carne bovina. Ministerio de Agricultura y Ganadería: Región Pacífico CentralMAG (en línea). San José, Costa Rica. 61 p. Consultado 07 ene. 2015. Disponible en http://www. mag.go.cr/bibliotecavirtual/a00050.pdf

Becerra, M. 2001. Consideraciones sobre estrategias sostenibles para el control de Fasciola hepatica en Latinoamérica. Revista Colombiana de Ciencias Pecuarias 14:228-235.

Bennema, S; Ducheyne, E; Vercruysse, J; Claerebout, E; Hendrickx, G; Charlier, J. 2011. Relative importance of management, meteorological and environmental 
factors in the spatial distribution of Fasciola hepatica in dairy cattle in a temperate climate zone. International Journal for Parasitology 41(2):225-33.

Chang, E; Cartín, M. 1983. Diagnóstico y control de la fasciolosis bovina en el distrito de Santa Cruz de Turrialba, provincia de Cartago. Tesis Lic. Heredia, Costa Rica, Universidad Nacional. 49 p.

Chavarría, R. 1939. Algunos parásitos de nuestro ganado de destace. Revista Centro Nacional de Agricultura IV (7/8/9):261-291.

Das Chagas, C; Batista, M; Vilhena, I; Rauta De Avelar, B; Salim, M; Molinari, D. 2011. Prevalence of liver condemnation due to bovine fasciolosis in Southern Espírito Santo: temporal distribution and economic losses. Revista Brasileria de Parasitologia Veterinária 20:49-53.

Díaz, A; Calvo, B; Trelles, S. 2013. La rastreabilidad del ganado bovino: La experiencia de Costa Rica Una decisión estratégica y sostenible. Documento elaborado en el marco del Proyecto: Desarrollo e Implementación de un Sistema de Control de Movimiento de Ganado Bovino en Costa Rica. IICA. San José, Costa Rica. 61 p.

DIPOA (Dirección de inocuidad de productos de origen animal, Costa Rica). 2012. Servicio Nacional de Salud Animal. Inspección Post mortem en Bovinos: DIPOA- PG-013 (en línea). Heredia, Costa Rica. 30 p. Consultado 15 abr. 2015. Disponible en http://www. senasa.go.cr/senasa/sitio/files/280612075654.pdf

Espinoza, J; Terashima, A; Herrera, P; Marcos, L. 2010. Fasciolosis humana y animal en el Perú: impacto en la economía de las zonas endémicas. Revista Peruana de Medicina Experimental y Salud Pública 27(4):604-612.

Fromsa, A; Meharenet, B; Mekibib, B. 2011. Major Trematode Infections of Cattle Slaughtered at Jima Municipality Abattoir and the Ocurrence of the Intermediate Host in Selected Water Bodies of the Zone. Journal of Animal and Veterinary Advances 10(12):1592-1597.

Gorman, T; Sánchez, R; Fredes, F; Alacaino, H. 1998. Inmunodiagnóstico de fascioliasis bovina mediante ELISA y Western Blot. Parasitología al Día 22:16-22.

Herrera, R. 1979. Diagnóstico de fasciolosis: estudio comparativo entre la intradermoreacción, exámen coproparasitológico y contrainmunoelectroforesis. Tesis Lic. Heredia, Costa Rica, Universidad Nacional. 19 p.

Howell, A. 2011. Snail-borne diseases in bovids at high and low altitude in Eastern Uganda: Integrated parasitological and malacological mapping. Thesis submitted in partial fulfilment of the requirements for the award of MSc in Biology and Control of Parasites and Disease Vectors. Liverpool School of Tropical Medicine. Liverpool, Reino Unido. 77 p.

Howell, A; Mugisha, L; Davies, J; Lacourse, E; Claridge, J; Williams, D; Kelly-Hope, L; Betson, M; Kabatereine,
N; Stothard, J. 2012. Bovine fasciolosis at increasing altitudes: parasitological and malacological sampling on the slopes of Mount Elgon, Uganda. Parasites and Vectors 5(1):196.

Kaplan, R. 2001. Fasciola hepatica: a review of the economic impact in cattle and considerations for control. Veterinary therapeutics 2(1):40-50.

Malone, J. 2002. Diseases of the Hepatobiliary System: liver flukes in ruminants. In Smith, BP (ed.). Large Animal Internal Medicine. Mosby, Missouri, USA. p. 805-808.

Martínez, J; Robles, D; Rojo, F; Martínez, M. 2012. Comparison of three different techniques to diagnose Fasciola hepatica infection in experimentally and naturally infected sheep. Veterinary parasitology 190(1-2):80-86.

Mas-Coma, S. 2004. Human fascoliasis: epidemiological patterns in human endemic areas of South America, Africa and Asia. Southeast Asian Journal Tropical Medicine and Public Health 35(1):1-11.

Monteiro, K; Arsénio De Fontes, A; Castillo, R; Fernández, O; Fonseca, O; Percedo, M. 2013. Prevalencia de hígados decomisados y pérdidas económicas por Fasciola sp,. en Huambo, Angola. Revista Salud Animal 35(2):89-93.

Morales, G; Pino, L. 2004. (Conferencia). Fasciola hepatica y Distomatosis hepatica bovina en Venezuela. Red de Helmintología de FAO para América Latina y el Caribe: Contribución a la Conferencia Electrónica 2004. 19 p.

Morales, I; Murillo, L. 2015. Análisis del Censo Ganadero 2014 para el Sector Ganadero. Departamento de Investigación y Divulgación de Corporación Ganadera (en línea). San José, Costa Rica. 39 p. Consultado 15 abr. 2015. Disponible en http:// corfoga.org/2012/wp-content/uploads/2013/07/ An\%C3\%A1lisis-del-Censo-Agropecuario-2015para-el-Sector-Ganadero.pdf

Morphew, R; Wright, H; La Course, E; Woods, D; Brophy, P. 2007. Comparative proteomics of excretorysecretory proteins released by the liver fluke Fasciola hepatica in sheep host bile and during in vitro culture ex host. Molecular and cellular proteomics 6(6):963-972.

OPS (Organización Panamericana de La salud, USA). 2003. Zoonosis y enfermedades transmisibles comunes al hombre y a los animales: parasitosis. 3 ed. Washington, D.C. USA. 423 p.

Poder Ejecutivo, Costa Rica. 2001. Decreto Ejecutivo $\mathrm{N}^{\circ}$ 29588 -MAG - S, Reglamento Sanitario y de Inspección Veterinaria de Mataderos, Producción y Procesamiento de Carnes. Costa Rica. Consultado 07 ene. 2015. Disponible en http://www.pgrweb. go.cr/scij/Busqueda/Normativa/Normas/nrm_texto_ completo.aspx?param1=NRTC\&nValor $1=1 \&$ nValor $2=46546 \&$ nValor $3=49201 \&$ strTipM=TC 
Rojas, D. 2015. Prevalencia de Fasciola hepatica en bovinos sacrificados en mataderos de clase A de Costa Rica durante el 2014. Tesis M. Sc. Heredia, Costa Rica, Universidad Nacional. $106 \mathrm{p}$.

Sanchez, M; Fraser, L. 2013. Investigating the impact of fasciolosis on cattle carcase performance. Veterinary Parasitology 193:307-311.

Schweizer, G; Braun, U; Deplazes, P; Torgerson, P. 2005. Estimating the financial losses due to bovine fasciolosis in Switzerland. Veterinary Record 157:188-193.
Tsegaye, B; Abebaw, H; Girma, S. 2012. Study on coprological prevalence of bovine fasciolosis in and around Woreta, Northwestern Ethiopia. Journal of Veterinary Medicine and Animal Health 4(7):89-92.

Vintimilla, N.2013.Prevalencia de parásitos gastrointestinales en bovinos que sacrifican en el Camal Municipal de Santa Isabel. Cuenca, Ecuador. Tesis Lic. Cuenca, Ecuador, Universidad de Cuenca. 160 p.

Yildirim, A; Ica, A; Duzlu, O; Inci, A. 2007. Prevalence and risk factors associated with Fasciola hepatica in cattle from Kayseri province, Turkey. Revue de Médecine Vétérinaire 158(12):613-617. 